\title{
The significance of aspects of screening for obstructive sleep apnoea in children with Down syndrome
}

\section{Keywords:}

Screening, obstructive sleep apnoea, Down syndrome

This is the peer reviewed version of the following article: Stores, R. J. and Stores, G. (2014), The significance of aspects of screening for obstructive sleep apnoea in children with Down syndrome. Journal of Intellectual Disability Research, 58: 381-392. doi: 10.1111/jir.12033, which has been published in final form at http://onlinelibrary.wiley.com/doi/10.1111/iir.12033/full. This article may be used for noncommercial purposes in accordance with Wiley Terms and Conditions for Self-Archiving. 


\section{Abstract}

\section{Background}

The sleep problems of children with intellectual disabilities remains a relatively neglected topic in spite of the consistent reports that such problems are common, often severe and persistent with potentially serious consequences for the children and their families. Children with Down syndrome (DS) are a case in point. They often suffer from obstructive sleep apnoea (OSA), early detection of which is recommended because of its potentially adverse effects on development. This study is concerned with aspects of assessment which have been considered important in helping to recognise OSA in children with DS. The relationships between different objective measures, and between these measures and parental reports of their child's sleep and daytime behaviour, were explored.

\section{Method}

Overnight recordings were carried out on a group of children with Down syndrome $(n=31)$ involving video and audio recording, oximetry and activity monitoring during sleep. Parents also completed questionnaires concerning their child's sleep and daytime behaviour.

\section{Results}

1. Parents' reports of restless sleep and noisy breathing were supported by objective measures of activity during sleep and audio recording respectively.

2. No significant association was found between objective measures of restlessness during sleep and 'snoring' (see later for definition), nor were objective measures of restlessness related to reductions in overnight blood oxygen levels. However, the objective measure of snoring was significantly associated with reductions in overnight blood oxygen levels.

3. All three of the objective measures were significantly associated with parental reports of various types of disturbed daytime behaviour.

\section{Conclusions}

The findings have implications for aspects of screening for OSA in children with DS and for the interpretation of the relevance of the results to the children's daytime behaviour. 


\section{Introduction}

The sleep problems of children with intellectual disabilities remains a relatively neglected topic in spite of the consistent reports that such problems are common, often severe and persistent with potentially serious effects on both the child and the family (Stores 2010). The present report focuses on an aspect of an aetiologically distinct group of such children, namely those with Down syndrome (DS). This group has been the subject of occasional sporadic surveys such as those by Breslin et al (2011), Carter et al (2009), Cotton \& Richdale (2006) and Stores \& Stores (2004). However, the details of the sleep disturbances associated with this condition, and the need for prompt recognition, warrant further investigation because of their relevance to the healthcare problems of children with DS (van GomerenOosterom et al 2011; McGrath et al 2011).

Collectively, these surveys indicate various types of sleep problems. As in children in general, sleeplessness is common and usually the result of failure to acquire good sleep habits for various reasons including parenting practices (Mindell et al 2006). Inadequate or poor quality sleep from whatever cause is likely to lead to daytime sleepiness and underperformance, or hyperactivity from being 'overtired' (Fallone et al 2002). Lesser mention is made of parasomnias (episodes of sleeprelated unusual behaviours, experiences or movements) although there is reason to expect that these are more common in children with DS than commonly supposed (Stores \& Stores in press). Early recognition and accurate diagnosis of the sleep disorders underlying these various sleep problems are important because of the improvements in learning and behaviour that can be expected following appropriate treatment (Richdale \& Wiggs 2005).

In these surveys, high rates of symptoms and signs suggestive of obstructive sleep apnoea (OSA) have been consistently reported by parents of children with DS. The Royal College of Paediatrics and Child Health SPARCDIC report (2009) suggests that fully diagnosed OSA is a complication in $50 \%$ or more of children with DS. Its many potentially serious cognitive, behavioural and other medical consequences, which have been summarised by Rosen (2011), are largely due to the disruptive effect of OSA on sleep ('fragmentation') which impairs its quality and restorative properties. In view of these adverse effects, the SPARCDIC report recommended regular screening of infants with Down syndrome at an early stage. Similar recommendations have been made by others (Rosen et al 2011; Primhak \& Kingshott 2012).

The question arises: What are the nature and implications of the variables that might constitute the screening procedure? Carroll and Loughlin (1995a) suggested guidelines for the evaluation of a child with breathing difficulties during sleep. Their basic screening variables included history of snoring, home audio taping and home overnight oximetry. On the other hand, Shott and colleagues (2006) recommended baseline polysomnography (PSG) for young children with DS on the grounds that parental impressions can be misleading. Either way, detailed diagnostic assessment is required when screening raises the possibility of OSA (Gozal \& Kheirandish-Gozal 2008). 
The focus of the present study concerns aspects of initial screening as suggested by Carroll and Loughlin, as distinct from PSG which involves more complicated assessment in hospital. In addition, as restless sleep (or 'bed thrashing') has been described on children with OSA (Carroll \& Loughlin 1995b), the nature and significance of this rather ill-defined phenomenon was considered.

The following three questions were addressed:

1. What is the relationship between parental reports of symptoms and signs in their child suggestive of OSA, and objective assessments of night-time restlessness, snoring and the changes of blood oxygen levels which have been associated with OSA .

2. What are the inter-relationships between these objectively measured parameters?

3. Are these three objectively assessed measures as indicators of quality of sleep, impairment of which is considered to be the cause of the impaired learning and behaviour associated with OSA (Owens 2009), linked to daytime behaviour problems as reported by parents?

\section{Methods}

\section{Participant recruitment}

Children with DS were recruited from the mailing list of an educational Down Syndrome trust which provided advice and support to families. An explanatory letter and information sheet were sent to parents with an invitation to take part in the survey. Further details were offered if required. The research was undertaken with the understanding and written consent of each child's parents and with the approval of the first author's research committee.

Assessments (conducted in the families' homes)

\section{General}

Characteristics including age, gender, type of schooling and basic medical information were assessed by personal interview and questionnaires.

\section{Subjective assessment of sleep (including restlessness and snoring)}

The sleep questionnaire (for completion by parents) used in the present study was that compiled by Simonds and Parraga (1982) which has been used to assess the sleep of other children with a developmental disorder (Johnson et al 2005). Twenty three items describe the child's general sleeping arrangements and habits, and 26 items concern the frequency of occurrence of various types of sleep disturbance. The sleep disturbance items can be grouped under four general headings: 
1. Disorders of initiating and maintaining sleep (6 items)

2. Features associated with obstructive sleep apnoea (6 items)

3. Other sleep disorders/behaviours occurring during sleep (8 items)

4. Sleep related disorders/behaviours occurring during the day (6 items)

Each item is rated as applying to the child over the previous 4 weeks on the following 6 point scale: never, less than once a month, about once a month, about once a week, several times a week, or daily.

\section{Objective measures of sleep: body movements during sleep}

Actometry records body movements. It has an established place in the assessment of children's sleep patterns (basically indicating periods of sleep or wakefulness) where the details of sleep physiology are not required (Sadeh 2011). A Gaehwiler Electronic (now Sing Electronic) Activity Monitor was used. This is a compact, wrist watch-like device which has no wires or leads. Its internal sensor triggers bodily movement and the signal is handled and scored by an internal microprocessor. The number of cumulated movements can be recorded over successive periods for as long as two weeks. A value between 0 (no movement) to 250 (maximum movement) is stored for each successive period. At the end of the recording period, the device is connected to a computer for the transfer of data. Analysis can then be performed and a graphic printout produced.

The activity variables used were based on those used by Aubert-Tulkens et al (1987) in their study of sleep apnoea using wrist activity monitoring. A Movement Index (MI) was obtained by dividing the number of 30 second periods with a value greater than 0 (i.e. with movement) by the total number of 30 second epochs. A Fragmentation Index (FI) was defined as the ratio of the number of 30 second immobility phases to the total number of immobility phases of all durations multiplied by 100 . Lastly, a Mean Activity Value (MAV) was calculated for each child. This was the mean activity value for each 30 second period across the sleep duration period. MI, FI and MAV were calculated for period from time asleep to time woke up (as defined by parental diaries and video recordings). Any periods of wakefulness were omitted from the analysis. The child's overall time spent asleep (Total Sleep Time) was also calculated from the parental diaries.

\section{Objective measurement of snoring during overnight sleep}

For the purposes of this study and reporting of the findings, the definition of snoring proposed by Lugaresi et al (1994) was used i.e. any clearly defined noise of a vibratory, gasping or snorting type appearing to occur intermittently on inspiration. This inclusive definition was considered appropriate in view of the variety of reported types of noisy breathing during sleep in children with OSA compared with adults (Carroll \& Loughlin 1995a). 
Video and audio recordings were carried out using an infra-red Sanyo camera which could record the children asleep overnight in complete darkness, a directional microphone and an Hitachi portable video cassette recorder/replay viewer. An eight hour recording was obtained for each child using a four hour tape and a long play facility. Although each recording did not extend to the full duration of the child's sleep, it was felt that the procedure adopted was the only practical way of obtaining overnight video/audio recordings in the circumstances and capable of providing an adequate and standard sample of sleep events. A time/date generator allowed the time and date to be displayed continuously on the screen for the purpose of data analysis. Each tape was viewed for the full eight hour recording period. An audio recording data sheet was compiled and each recording was coded for snoring. Each 30 second epoch was coded as either 'snoring' or 'not snoring'. Percentage of 30 second epochs scored as snoring was then computed from time asleep to time woke up for each child separately. Any periods of wakefulness were omitted from the analysis.

\section{Oximetry}

The Oxford MPA II system (Oxford Medical Systems) with a Novametrix pulse oximeter (Novametrix Medical Systems) was used to obtain continuous overnight measurements of arterial oxygen saturation. This system records data onto a standard audio cassette tape. Data analysis was carried out using the Oxford Medilog 9000 system (Oxford Medical Systems). A printout contained the following information:

- recording start and end times

- total number of dips in oxygen saturation greater than $4 \%$ from baseline

- desaturation index (defined as number of $>4 \%$ dips per hour for recording period)

- maximum oxygen saturation

- minimum saturation during recording

An hypoxic-dip event log was also obtained which contained time of each event, duration of each event (in seconds) and minimum oxygen saturation during each event. In addition, a plot was obtained showing oxygen measurements throughout the recording period.

\section{Measures of daytime behaviour}

Two separate behavioural rating scales were completed by parents:

\section{Aberrant Behavior Checklist ( $A B C$ ) (Community version)}

This scale was developed specifically for people with learning difficulties, including children (Aman, Singh, Stewart \& Field, 1985) and has been used effectively in studies of patients with DS (Capone et al 2006).

The $A B C$ is a 58 item questionnaire for rating 'inappropriate and maladaptive behaviour' on the following subscales based on factor analysis (Marshburn \& Aman, 1992): 
1. Irritability (15 items): irritability, agitation and crying

2. Lethargy (16 items): lethargy and social withdrawal

3. Stereotypies ( 7 items): stereotypic behaviour i.e. repetitive semi-purposeful behaviours

4. Hyperactivity (16 items): hyperactivity and non-compliance

5. Inappropriate speech (4 items): excessive and repetitive talking

In the present study, a total daytime behaviour problem score was calculated. This was the sum of all five subscales.

Parents rate the child over the previous four weeks on each items using a four point scale:

$0=$ not at all a problem

$1=$ the behaviour is a slight problem

$2=$ the problem is moderately serious

$3=$ the problem is severe

The questionnaire takes about 10 minutes to complete. It has been shown to have satisfactory psychometric properties (Aman \& Singh, 1986).

\section{Conners Parent Rating Scale (48-item version)}

The Conners Parent Rating Scale has been widely used for assessing behaviour problems in children. This 48-item version yields 5 factors (Goyette, Conners \& Ulrich, 1978):

\section{Conduct problem \\ 2. Learning problem \\ 3. Psychosomatic \\ 4. Impulsive-hyperactive \\ 5. Anxiety.}

It was considered that this factor structure appropriately complemented that of the ABC.

Symptoms are rated on a 4-point scale (0-3). Parents are asked to rate their child on each item as 'not at all a problem', 'just a little', 'pretty much' or 'very much'. Specific item responses are summed to give the 5 factor scores. Adequate reliability and validity has been reported (Conners, 1990).

\section{Procedure}

\section{Overnight recording}

On the night of the recording, the families were visited by the first author and the procedure was explained to the parents and child. They were shown the equipment and time was spent familiarising the child with each piece of equipment. If the child was not ready for bed, the parents were shown how to start the video recorder and attach the activity monitor and the oximeter later in the evening. 


\section{Video and audio recording}

The infra-red camera, microphone and portable video cassette recorder were set up in the child's bedroom and the parents were told how to start recording either when the child got into bed or a short while after they had fallen asleep. Parents were asked to make sure their child was in view using the built-in display on the recorder. Parents were also instructed on how to turn off the display so that the light from the display would not disturb or attract the child's attention during the night. Parents were told that the recorder would switch itself off when the recording had finished.

\section{Activity monitor}

Each child was shown the activity monitor and told they were to wear it when they were in bed asleep. The non-dominant hand was used. If parents were not sure which was the non-dominant hand, the left hand was used. If the child did not want the activity monitor to be put on when they were still awake, parents were asked to attach it once the child had fallen asleep. If the child was initially reluctant to try on the equipment, parents and/or brothers and sisters were asked to do so to encourage the child. A 'fake' activity monitor was also used for brothers and sisters to wear, partly to encourage the child with Down syndrome to wear the real monitor and partly to reduce the likelihood of brothers or sisters feeling left out. The activity monitor was kept on all night and removed once the child got up in the morning. If parents were aware of the monitor being removed during the night, they were asked to replace it. They were also asked to make a note of the time put on, time removed, time into bed etc. using the diary provided.

\section{Diary}

Parents were asked to complete a diary on the night of the recording indicating the following:

- time activity monitor put on

- time into bed

- time went to sleep

- time and length of any waking during the night of which parents were aware

- time woke up in morning

- time got up in morning

- time activity monitor taken off in morning

- any periods activity monitor was removed during the night of which parents were aware (time removed and time replaced).

\section{Oximetry}

The oximeter sensor was attached to the child's toe when the child got into bed either by the first author or by parents after instruction, and covered with a sock. As with the activity monitor, if the child did not want the probe attached when they were awake, parents were asked to attach it once the child had fallen asleep. However, all the children were shown the probe beforehand so that they were familiar with the equipment if they woke up in the night and found it attached. Once the probe was 
attached, the lead was fed out of the bottom of the bed. If removed during the night, parents were asked to replace it and instructed on how to do so. If the child would not allow the parents to attach the sensor, parents were told to leave it off, but to continue with the other measures.

Parents were provided with an instruction sheet which covered the whole procedure. They were also given the first author's telephone number and told to ring at any time, day or night, if they encountered any problems.

Parents were also asked to complete the sleep questionnaire on the night of the overnight recording.

\section{Daytime behaviour}

On the morning following the overnight recordings and completion of the sleep questionnaire, the child's primary caregiver (the mother in all cases) was asked to complete the daytime behaviour questionnaires.

\section{Data analysis}

Analysis was performed using SPSS. Statistical tests used were t-tests and Pearson's correlation coefficients. 


\section{Results}

\section{General characteristics}

Table 1 shows the total number, gender, age, type of school attended and, as provided by parents, basic medical information for the sample.

\section{INSERT TABLE 1}

\section{Representative nature of the sample}

The current sample was found to be very similar with regards to their general characteristics, sleeping arrangements and habits, and frequency of sleep disorders and behaviours, to a larger sample of 91 children with DS in a separate study (Stores et al 1996) suggesting they were representative of this larger sample and not subject to selection bias.

\section{Sleep questionnaire information}

\section{Sleeping arrangements \& habits, sleep quality, and total sleep time}

Information was collected on the children's sleeping arrangements, bedtime routines, use of transitional objects, sleep quality in terms of apparent value of the sleep obtained, whether or not parents had received previous advice for a sleep problem and whether or not they considered their child had a sleep problem and total sleep time. This information is summarised in Table 2.

\section{INSERT TABLE 2}

\section{Sleep disturbances}

Table 3 gives an indication of the relative occurrence of sleep disturbances considered to be clinically significant ie. reported by parents to be occurring frequently (daily or several times a week). These disturbances are grouped as problems of initiating and maintaining sleep, features associated with obstructive sleep apnoea, other disturbances during sleep, and sleep related disturbances occurring during the day.

\section{INSERT TABLE 3}




\section{Parental reports of restless sleep and objective measures from actometry recordings}

Activity monitor scores were compared for children rated as frequently restless by parents (i.e. several times a week or daily) and children not rated as frequently restless by parents (i.e. never, less than once a month, about once a month or about once a week). The results are shown in Table 4.

\section{INSERT TABLE 4}

The group rated as restless scored higher than the group not rated as restless on all three activity monitor scores. However, this difference reached statistical significance with the MI score only.

\section{Parental reports of snoring and objective measures using audio recordings}

The percentage of epochs scored as snoring from the audio tapes were compared for children rated as frequent snorers by parents (i.e. several times a week or daily) and children not rated as frequent snorers by parents (i.e. never, less than once a month, about once a month or about once a week). The results are shown in Table 5.

\section{INSERT TABLE 5}

The group rated as frequent snorers had a significantly higher percentage of epochs scored as snoring than the non-snorers.

Therefore, parents' reports of restless sleep and snoring were supported by objective measures of activity during sleep and audio recording respectively.

\section{Associations between objective measures of restless sleep, snoring and blood oxygen saturation during sleep}

Associations between the objective measures of activity, snoring and oximetry were explored using Pearson's correlation coefficients. The results are shown in Table 6.

\section{INSERT TABLE 6}

No significant association was found between objective measures of restlessness during sleep and snoring. Similarly, there was no significant association between objective measures of restlessness 
and repeated significant reductions in overnight blood oxygen levels. However, a significant association was found between the objective measure of snoring and repeated significant reductions in overnight blood oxygen levels. MI was significantly correlated with $\mathrm{FI}$ and mean activity value

\section{Associations between total sleep time, objective measures of activity, snoring and blood oxygen saturation during sleep and parents' ratings of daytime behaviour}

Associations between the total sleep time, objective measures of activity, snoring and oximetry and parents' ratings of daytime behaviour were explored using Pearson's correlation coefficients. The results for the Aberrant Behavior Checklist are shown in Table 7 and the Conners Rating Scale in Table 8.

\section{INSERT TABLE 7}

Total sleep time was significantly negatively correlated with parental reports of lethargy, stereotypies, inappropriate speech and total $A B C$ score. The Mean Activity Value was significantly associated with parental reports of inappropriate speech. Percentage of epoch scored as snoring was significantly correlated with lethargy and desaturation index was significantly correlated with inappropriate speech.

\section{INSERT TABLE 8}

Total sleep time was significantly negatively correlated with parental reports of conduct disorder, impulsivity-hyperactivity and anxiety. The Movement Index was significantly associated with parental reports of impulsivity-hyperactivity and anxiety and the Fragmentation Index was significantly associated with impulsivity-hyperactivity. The Mean activity value was significantly associated with conduct disorder. 


\section{Discussion}

The main findings of this exploratory study were as follows:

1. Good correspondence was apparent between parental reports of restlessness and noisy breathing during sleep and objective measures of activity and audio recording results respectively.

2. No significant association was found between objective measures of restlessness during sleep and snoring, nor were objective measures of restlessness related to reductions in overnight blood oxygen levels. However, the objective measure of snoring was significantly associated with reductions in overnight blood oxygen levels.

3. All three of the objective measures were significantly associated with parental reports of various types of disturbed daytime behaviour.

A number of issues, relevant to further work in this field, arise from the nature of the present study.

(a) Choice of objective variables assessed.

As mentioned earlier, routine screening for OSA in children with DS has been recommended because of its common occurrence in such children and its possible harmful effects on their development.

Some accounts of childhood OSA have emphasised parental reports of snoring and restless sleep as important possible indicators of OSA (Carroll \& Loughlin 1995b). This is in contrast to actual apnoeic episodes which, although an important feature of adult OSA, are not necessarily prominent in children with the condition (Marcus 2000). Some support for the screening value of snoring and restless sleep was provided by the results of a factor analysis of clinical features linked to OSA in children with DS (Stores et al 1998). Overnight oximetry was included in the present study because its significance in children with suspected OSA did not seem to have been systematically investigated (Carroll \& Loughlin 1995b).

(b) Recording and analysis of objective variables.

As mentioned earlier, for present purposes, snoring was defined as any clearly defined noise of a vibratory, gasping or snorting type occurring intermittently on inspiration (Lugaresi et al 1994). This includes various types of noisy breathing including gasping or snorting. Clearly, these judgments involve some degree of subjectivity and ideally independent inter-rater reliability would have been determined. For further studies, an automated audio recognition and scoring system could help to improve accuracy and significantly shorten the time required for the manual analysis that was employed. 
That only a single night of recording was used raises the possibility of 'first night effects' which, however, have been reported as not a problem with home actometry recordings in children (Sadeh 2011). Nevertheless, if possible, more than one night of recording is advisable.

(c) What reliance can be placed on parental reports of features that might suggest that their child suffers from OSA?

In routine clinical practice, information about children's sleep has to be based primarily on parental reports. However, such reporting might be inaccurate because parents may not be well placed to observe their child at night, or they may be unaware of what is usual or otherwise about children's sleep (Rosen et al 2011). The accuracy of parental reporting has been the subject of little research and opinions have differed. Acebo and colleagues (1994) reported a strong relationship between mothers' reports about timings of their children's sleep and actometry results. On the other hand, Marcus et al (1991) suggested that parents' reports may not be a good guide to the occurrence of OSA in children with DS. The present findings suggest that information from parents can be useful in initial enquiries for screening purposes.

(d) The significance of the objective measures considered separately.

Although restless sleep (or 'bed thrashing') has been considered a major feature of childhood OSA (Carroll \& Loughlin 1995b), in the present study restlessness was not significantly associated with the other two objective measures. It may be that some children with DS have restless sleep for reasons other than OSA. Stores et al (1998) suggested that restlessness is significantly associated with repeated night-time waking which could at least be partly the result of a behavioural sleep disorder, and Levanon et al (1999), on the basis of polysomnographic findings, has described sleep fragmentation (possibly linked to restlessness) in children with DS not wholly attributable to OSA. There is a need for more refined analysis of 'restless sleep' and the particular aspects of sleep, including respiratory abnormalities, associated with it.

In the present study, recorded snoring was significantly associated with reductions in overnight blood oxygen levels. This lends support to the view that such noisy breathing alone can be associated with disordered sleep physiology (Carroll \& Loughlin 1995b).

The mean oxygen desaturation index of this group of children with DS was significantly more marked than normative values for the children in general. According to the normative data published by Marcus et al (1992), about $25 \%$ of the children with DS in the current sample showed an abnormally high desaturation index. This raises concerns about the respiratory adequacy of these children (whatever the precise diagnosis might be achieved by full diagnostic assessment) and the potentially harmful effects this may have on these children. 
(e) Possible associations between the objective measures and parental reports of their children's behaviour.

This issue concerns whether the objective measures (as possible indicators of impaired quality of sleep) might be predictive of impaired psychological function during the day.

All three variables were associated with behavioural disturbances but a coherent account of the findings, taking each sleep variable separately, is difficult because of the number and variety of the psychological measures taken and the relatively limited overall sample size. That said, the results indicate that more extensive and focussed studies of the relationships between the sleep variables and psychological assessments might well be instructive.

(f) Feasibility of such screening assessments.

The overnight measures used in the present study proved feasible and largely acceptable to the children and their families. This is likely to be mainly attributable to the efforts made (as detailed in the Procedure section of this paper) to carefully explain and introduce the assessment procedures to the children and their parents. Performing the recordings and other assessments in the home environment is also likely to have helped. Satisfactory audio-visual recordings were obtained in $81 \%$ of the children (failures were the result of equipment problems), and actometry was successful in $78 \%$. Oximetry measures were obtained in $65 \%$, failures being the child not tolerating the attachments or malfunction of the equipment. More time spent familiarizing the children with the procedure and use of more recently developed recording systems would be likely to improve the success rate with this particular procedure.

(g) Other considerations.

The exploratory nature of this study has been emphasised. Clearly, there is a need to assess the consistency of the findings by further studies on larger samples of children with DS using the latest forms of appropriate technology. 


\section{References}

Acebo C., Sadeh A., Seifer, R., Tzischinsky., Dickstein., Aytur, S. et al. (1994) Mothers' assessment of sleep behaviours in young children: scale reliability and validation versus actigraphy. Sleep Research 23, 96.

Aman M. G., Sing N. N., Stewart, A. W. \& Field, C. J. (1985) The Aberrant Behavior Checklist: a behaviour rating scale for the assessment of treatment effects. American Journal of Mental Deficiency 89, 485-91.

Aman M. G. \& Singh N. N. (1986) Aberrant Behavior Checklist Manual. Slosson Educational Publications, New York.

Aubert-Tulkens G., Culee C., Harmant-Van Rijckevorsel \& Rodenstein D. O. (1987) Ambulatory evaluation of sleep disturbance and therapeutic effects in sleep apnea syndrome by wrist activity monitoring. American Review of Respiratory Diseases 136, 851-6.

Breslin J.H., Edgin J.O., Bootzin R.R., Goodwin J.L. \& Nadel L. (2011) Parental reports of sleep problems in Down syndrome. Journal of Intellectual Disability Research 55, 1086-91.

Capone G., Goyal P., Ares W. \& Lannigan E. (2006) Neurobehavioral disorders in children, adolescents, and young adults with Down syndrome. American Journal of Medical Genetics C Seminars in Medical Genetics 142C, 158-72.

Carroll J. L. \& Loughlin G. M. (1995a) Obstructive sleep apnea in infants and children: clinical features and pathophysiology. In: Principles and Practice of Sleep Medicine in the Child (eds R. Ferber \& M. Kryger M.), pp. 163-91. Saunders, Philadelphia.

Carroll J. L. \& Loughlin G. M. (1995b) Obstructive sleep apnea in infants and children: diagnosis and management. In: Principles and Practice of Sleep Medicine in the Child (eds R. Ferber \& M. Kryger M.), pp. 193-216. Saunders, Philadelphia.

Carter M., McCaughey E., Annaz D. \& Hill C.M. (2009) Sleep disorders in a Down syndrome population. Archives of Disease in Childhood 94, 308-310.

Conners C. K. (1990) Conners Rating Scales Manual. Multi-Health Systems, Inc, Canada.

Cotton S. \& Richdale A. (2006) Brief report: parental descriptions of sleep problems in children with autism, Down syndrome and Prader-Willi syndrome. Research into Developmental Disabilities 27, $151-61$. 
Fallone G., Owens J. A. \& Deane J. (2002) Sleepiness in children and adolescents: clinical implications. Sleep Medicine Reviews 6, 287-306.

Goyette C. H., Conners C. K. \& Ulrich R. F. (1978) Normative data on revised Conners parent and teacher rating scales. Journal of Abnormal Child Psychology 6, 221-36.

Gozal D. \& Kheirandish-Gozal L. (2008) The multiple challenges of obstructive sleep apnea in children: morbidity and treatment. Current Opinion in Pediatrics 20,654-8.

Johnson H., Wiggs L., Stores G.\& Huson S. (2005) Psychological disturbance and sleep disorders of children with neurofibromatosis type 1. Developmental Medicine and Child Neurology 47, 237-42.

Lugaresi E., Cirignotta F., Montagna P. \& Sforza E. (1994) Snoring: pathogenic, clinical and therapeutic aspects. In: Principles and Practice of Sleep Medicine (eds M.H. Kryger, T. Roth, T. \& W.C. Dement), p 621. Saunders, Philadelphia.

Marcus C. L., Keens T. G., Bautista D. B., von Pechman W. S. \& Ward S. L. (1991) Obstructive sleep apnea in children with Down syndrome. Pediatrics 88, 132-9.

Marcus C. L., Omlin K.J., Basinski D. J., Bailey S. L., Rachel A. B., Von Pechmann WS et al. (1992) Normal polysomnographic values for children and adolescents. American Review of Respiratory Disease 146, 1235-9.

Marcus C.L (2000) Obstructive sleep apnea syndrome: differences between children and adults. Sleep 23, S140-1.

Marshburn E. C. \& Aman M. G. (1992) Factor validity and norms for the Aberrant Behavior Checklist in a community sample of children with mental retardation. Journal of Autism and Developmental Disorders 22, 357-73.

McGrath R.J., Stransky M.L., Cooley C. \& Moeschler J.B. (2011) National profile of children with Down syndrome: disease burden, access to care, and family impact. Journal of Pediatrics 159, $535-40$.

Mindell JA, Kuhn B, Lewin DS, Meltzer LJ \& Sadeh A. (2006) Behavioral treatment of bedtime problems and night wakings in infants and young children. Sleep 29, 1263-76.

Owens J.A. (2009) Neurocognitive and behavioural impact of sleep disordered breathing in children. Pediatric Pulmonology 44, 417-22. 
Primhak R. \& Kingshott R. (2012) Sleep physiology and sleep-disordered breathing: the essentials. Archives of Disease in Childhood 97, 54-8.

Richdale A. \& Wiggs L. (2005) Behavioral approaches to the treatment of sleep problems in children with developmental disorders: what is the state of the art? International Journal of Behavioral and Consultation Therapy 1, 165-89.

Rosen D. (2011) Management of obstructive sleep apnea associated with Down syndrome and other craniofacial dysmorphologies. Current Opinion in Pulmonary Medicine 17, 431-6.

Rosen D., Lombardo A., Skotko B. \& Davidson E.J. (2011) Parental perceptions of sleep disturbances and sleep-disordered breathing in children with Down syndrome. Clinical Pediatrics (Philadelphia) 50, 121-5.

Royal College of Paediatrics and Child Health (2009) Working party on sleep physiology and respiratory control disorders in childhood. Standards for children with disorders of sleep physiology (SPARCDIC Report). RCPCH, London.

Sadeh A. (2011) The role and validity of actigraphy in sleep medicine: an update. Sleep Medicine Reviews 15, 259-67.

Shott SR, Amin R, Chini B, Heubi C, Hotze S \& Akers R. (2006) Obstructive sleep apnea: should all children with Down syndrome be tested? Archives of Otolaryngology and Head and Neck Surgery 132, 432-6.

Simonds J. F. \& Parraga H. (1982) Prevalence of sleep disorders and sleep behaviours in children and adolescents. Journal of the American Academy of Child Psychiatry 21, 383-8.

Stores G. (2010) Sleep in developmental disorders. In: Foundations of Psychiatric Sleep Medicine (eds JW Winkelman \& DT Plante), pp 371-86. Cambridge University Press, New York.

Stores R., Stores G., Buckley S. \& Fellows B. (1996) The pattern of sleep problems in children with Down's syndrome and other intellectual disabilities. Journal of Applied Research in Intellectual Disabilities 9, 145-58.

Stores R., Stores G., Buckley S. \& Fellows B. (1998) A factor analysis of sleep problems and their psychological associations in children with Down's syndrome. Journal of Applied Research in Intellectual Disabilities 11, 345-54. 
Stores R. \& Stores G. (2004) Evaluation of group-administered instruction for parents to prevent or minimize sleep problems in young children with Down syndrome. Journal of Applied Research in Intellectual Disabilities 17, 61-70.

Stores G \& Stores R. Review: Sleep disorders and their clinical significance in children with Down syndrome. Developmental Medicine and Child Neurology. In press.

van Gomeren-Oosterom H.B.M., Fekkes M., Buitendijk S.E., Mohangoo A.D., Bruil J. \& Van Wouwe J.P. (2011) Development, problem behavior, and quality of life in a population based sample of eightyear-old children with Down syndrome. PLoS One 2011; 6: e21879 DOI: 0.1371/journal.pone.0021879 
Table 1: General characteristics of the sample

$\mathbf{N}$

\section{Gender}

Boys

Girls

Age (years)

Mean

S.D.

Range

\section{Schools}

Mainstream

Special

Split placement

Nursery

\section{Medical Information}

Usually suffers from coughs and colds

Heart problems

Adenoids and tonsils removed
31

$15(48 \%)$

$16(52 \%)$

8.7

3.3

$2.3-16.3$

$20(65 \%)$

$8(26 \%)$

$1(3 \%)$

$2(7 \%)$

$21(68 \%)$

13 (42\%)

$11(36 \%)$ 
Table 2: Sleeping arrangements \& habits, sleep quality, and total sleep time

$\%$

\section{Arrangements}

Has own room

Usually sleeps on own

Has bedtime routine

97

Has transitional object

Sleep quality

Is usually a light sleeper

Wakes up in morning refreshed

Wakes up in morning in a bad mood

If has poor night sleep, acts differently next day

Average total sleep time (hours)

Mean

S.D.

Range 


\section{Disorders of initiating and maintaining sleep}

\section{Settling}

Waking

Early waking

Reluctant to go to bed

nsists on sleeping with someone

Reluctant to go to bed due to fears

Features associated with obstructive sleep apnoea

Difficulty breathing

Restless

Snores loudly

Unusual positions

Sleeps with neck extended

Mouth breathing

Apnoeic episodes

Gags/chokes

Headaches on awakening

Sweats a lot

Kicking and flailing

\section{Other sleep disturbances}

Sleep talking

Sleep walking

Teeth grinding

Head banging

Body rocking

Tongue biting

Nightmares

Night terrors

Bed wetting

Sleep related disturbances during the day

Excess daytime sleepiness

Difficulty staying awake

Falls asleep

Lacks energy

Irritable

Miserable

Floppy attacks

Attacks of inability to move

Irresistible sleep attacks

Drowsy

Daytime overactivity

Daytime naps 
Table 4: Actometry values for children rated as restless and not restless by parents

\begin{tabular}{||l||c|c|c|c||}
\hline & $\begin{array}{c}\text { Not restless } \\
(\mathbf{n}=9)\end{array}$ & $\begin{array}{c}\text { Restless } \\
(\mathbf{n}=15)\end{array}$ & $\mathbf{t}$ & $\begin{array}{c}\mathbf{p} \\
(\text { 1-tailed })\end{array}$ \\
\hline \hline $\begin{array}{l}\text { Movement Index } \\
\text { (MI) }\end{array}$ & 8.84 & 11.26 & 2.16 & $<0.01$ \\
\hline $\begin{array}{l}\text { Fragmentation Index } \\
\text { (FI) }\end{array}$ & 8.34 & 12.21 & 1.41 & $\mathrm{NS}$ \\
\hline $\begin{array}{l}\text { Mean Activity Value } \\
\text { (MAV) }\end{array}$ & 1.28 & 1.40 & 0.38 & $\mathrm{NS}$ \\
\hline
\end{tabular}


Table 5: Percentage epochs scored as snoring for children rated as frequent snorers and nonfrequent snorers by parents

\begin{tabular}{||l||c|c|c|c||}
\hline & $\begin{array}{c}\text { Non-snorers } \\
(\mathbf{n}=17)\end{array}$ & $\begin{array}{c}\text { Frequent } \\
\text { Snorers } \\
(\mathbf{n}=9)\end{array}$ & $\mathbf{t}$ & $\begin{array}{c}\mathbf{p} \\
(\mathbf{1}-\text { tailed })\end{array}$ \\
\hline \hline $\begin{array}{l}\% \text { epochs scored as } \\
\text { snoring }\end{array}$ & 3.04 & 7.56 & 2.10 & $<0.01$ \\
\hline
\end{tabular}


Table 6: Pearson's correlation coefficients for objective overnight measures

\begin{tabular}{||l||c|c|c|c|c||}
\hline & MI & FI & MAV & $\begin{array}{c}\text { \% epochs } \\
\text { scored as } \\
\text { snoring }\end{array}$ & $\begin{array}{c}\text { Desaturation } \\
\text { Index }\end{array}$ \\
\hline \hline MI & - & - & & & \\
\hline FI & $.7585^{* * *}$ & - & & & \\
\hline MAV & $.6196^{* * *}$ & .2379 & - & & \\
\hline $\begin{array}{l}\text { \% epochs } \\
\text { scored as } \\
\text { snoring }\end{array}$ & -.0981 & .0452 & -.3232 & - & \\
\hline $\begin{array}{l}\text { Desaturation } \\
\text { Index }\end{array}$ & -.0541 & -.0434 & -.1385 & $.5107^{*}$ & - \\
\hline \hline
\end{tabular}

${ }^{*}<0.05^{* *}<0.01^{* * *}<0.001$ (1-tailed)

Key:

$\mathrm{MI}=$ Movement index from activity monitoring

$\mathrm{FI}=$ Fragmentation index from activity monitoring

$\mathrm{MAV}=$ Mean Activity Value from activity monitoring

$\%$ epochs scored as snoring from audio recording

Desaturation index from oximetry 
Table 7: Pearson's correlation coefficients for objective overnight measures and Aberrant Behavior Checklist scores

\begin{tabular}{||l||c|c|c|c|c|c||}
\hline & Irritability & Lethargy & Stereotypies & Hyperactivity & $\begin{array}{c}\text { Inappropriate } \\
\text { speech }\end{array}$ & Total ABC \\
\hline \hline TST & -.2874 & $-.3423^{\star}$ & $-.4012^{\star}$ & -.2772 & $-.4787^{\star *}$ & $-.5304^{\star *}$ \\
\hline MI & -.0154 & -.3128 & -.1879 & .1836 & .0690 & -.0715 \\
\hline FI & -.1221 & -.2468 & -.0478 & .0780 & .0291 & -.1068 \\
\hline MAV & .3187 & -.0164 & -.2476 & .2171 & $.3545^{\star}$ & .1805 \\
\hline $\begin{array}{l}\text { \% } \\
\text { epochs } \\
\text { snoring }\end{array}$ & -.0358 & $.4239^{\star}$ & .3029 & .0198 & -.1511 & .1917 \\
\hline $\begin{array}{l}\text { Desat. } \\
\text { Index }\end{array}$ & -.2262 & -.2909 & -.1800 & .0132 & $.4693^{\star}$ & -.1575 \\
\hline
\end{tabular}

${ }^{*}<0.05^{* *}<0.01^{* * *}<0.001$ (1-tailed)

Key:

TST $=$ Total sleep time from sleep diaries

$\mathrm{MI}=$ Movement index from activity monitoring

$\mathrm{FI}=$ Fragmentation index from activity monitoring

MAV = Mean Activity Value from activity monitoring

$\%$ epochs scored as snoring from audio recording

Desat. index = Desaturation index from oximetry 
Table 8: Pearson's correlation coefficients for objective overnight measures and Conners Rating Scale (Parent) scores

\begin{tabular}{||l||l|l|l|l|l||}
\hline \hline & $\begin{array}{l}\text { Conduct } \\
\text { disorder }\end{array}$ & $\begin{array}{l}\text { Learning } \\
\text { problem }\end{array}$ & $\begin{array}{l}\text { Psychosoma } \\
\text { tic }\end{array}$ & $\begin{array}{l}\text { Impulsive- } \\
\text { Hyperactive }\end{array}$ & Anxiety \\
\hline \hline TST &.$- .4557^{\star \star}$ & -.1361 & .1175 & $-.3389^{\star}$ & $-.5061^{\star \star}$ \\
\hline MI & .1730 & -.2195 & -.0617 & $.3475^{\star}$ & $-.3694^{\star}$ \\
\hline FI & -.0472 & -.0790 & -.1750 & $.4088^{\star}$ & -.3209 \\
\hline MAV & $.4822^{\star *}$ & -.1709 & -.0164 & .1368 & -.2256 \\
\hline $\begin{array}{l}\text { \% epochs } \\
\text { snoring }\end{array}$ &.- .1672 & -.0568 & -.03014 & .0158 & .3353 \\
\hline \begin{tabular}{l} 
Desat. Index \\
\cline { 2 - 6 }
\end{tabular} &.- .0624 & -.2435 & -.1152 & .1041 & .1823 \\
\hline
\end{tabular}

${ }^{*}<0.05^{* *}<0.01^{* * *}<0.001$ (1-tailed)

Key:

TST = Total sleep time from sleep diaries

$\mathrm{MI}=$ Movement index from activity monitoring

$\mathrm{FI}=$ Fragmentation index from activity monitoring

$\mathrm{MAV}=$ Mean Activity Value from activity monitoring

$\%$ epochs scored as snoring from audio recording

Desat. index = Desaturation index from oximetry 